\title{
The clinical outcomes of late preterm infants: a multi-center survey of Zhejiang, China*
}

Xiaolu Ma1, Chunxiang Huang'2, Shouzeng Lou ${ }^{3}$, Qin $\mathbf{L v}^{4}$, Weidong Su${ }^{5}$, Jiarong Tan ${ }^{6}$, Youcheng Wang $^{7}$, Xinxin Wang ${ }^{8}$, Mingyuan $\mathrm{Wu}^{9}$, Ting $\mathrm{Xu}^{10}$, Manli Zhuang ${ }^{11}$, Lizhong $\mathrm{Du}^{1, * *}$ and the Provincial Collaborative Study Group for Late-preterm Infants

${ }^{1}$ Children's Hospital of Zhejiang University School of Medicine, Hangzhou, Zhejiang, China

${ }^{2}$ Quzhou Women and Children's Hospital, Quzhou, Zhejiang, China

${ }^{3}$ Fuyang Women and Children's Hospital, Fuyang, Zhejiang, China

${ }^{4}$ Ningbo Women and Children's Hospital, Ningbo, Zhejiang, China

${ }^{5}$ The Second People's Hospital of Wenzhou, Wenzhou, Zhejiang, China

${ }^{6}$ Huzhou Women and Children's Hospital, Huzhou, Zhejiang, China

${ }^{7}$ The People's Hospital of Jinhua, Jinhua, Zhejiang, China

${ }^{8}$ Zhejiang Provincial People's Hospital, Hangzhou, Zhejiang, China

${ }^{9}$ Women's Hospital of Zhejiang University School of Medicine, Hangzhou, Zhejiang, China

${ }^{10}$ Jinhua Central Hospital, Jinhua, Zhejing, China

${ }^{11}$ Zhoushan Women and Children's Hospital, Zhoushan, Zhejiang, China

\footnotetext{
Abstract

Objective: To explore birth rate, delivery mode, medical problems, requirement of respiratory support, and acute outcomes of late preterm infants in Zhejiang province in eastern China.

Methods: Eleven tertiary hospitals were recruited. Clinical data of every nursery admission from January to December 2007 were collected and analyzed.

*This study was supported by the National Natural Science Foundation of China (grant number: 30711120575 and 30672265).

${ }^{* *}$ Corresponding author:

Lizhong Du, PhD

Children's Hospital of Zhejiang University School of Medicine Hangzhou

Zhejiang

China

E-mail: dulizhong@yahoo.com.cn
}

Results: During the study period, 44,362 infants were born with an overall preterm birth rate of $8.9 \%$, and late preterm birth rate of $6.2 \%$. Late preterm infants had higher cesarean section rate than the whole population (64.9\% vs. $58.2 \%)$. One-fifth of the nursery admissions were late preterm infants, of whom, $63.8 \%$ were delivered by cesarean section. Respiratory distress $(42.1 \%)$ was the most common medical problem of late preterm infants. Hyperbilirubinemia (17.6\%), hypoglycemia (8.7\%) and sepsis (5.9\%) were also common. The first three primary diagnoses of respiratory distress included pneumonia (39.5\%), transient tachypnea of newborn (TTN) $(22.5 \%)$ and respiratory distress syndrome (RDS) (19.0\%). Compared with term infants, late preterm infants with respiratory distress needed more respiratory support with nasal continuous positive airway pressure (nCPAP) ( $21.4 \%$ vs. $11.6 \%)$ or with a mechanical ventilator $(15.4 \%$ vs. $11.0 \%)$, and also had higher in-hospital mortality (0.8\% vs. $0.4 \%)$.

Conclusions: Late preterm infants are associated with very high cesarean section rate and have more medical problems and poorer short-term outcomes than term infants in China.

Keywords: Cesarean section; late preterm; outcome; respiratory distress syndrome; transient tachypnea.

\section{Introduction}

In recent years, a subgroup of more mature preterm infants, born at $34 \% / 7$ to $36 \% / 7$ weeks' gestation, so-called "late preterm" infants, has become a focus of increased interest. Martin et al. [6] reported that during the last 15 years, the proportion of late preterm births in all US births increased from $7.3 \%$ in 1990 to $9.1 \%$ in 2005 , and this population accounted for $>70 \%$ of all preterm births $[3,6]$.

Late preterm infants are physiologically immature. Some authors [2, 5, 9] have documented that this group of infants have higher incidence of medical complications, neonatal intensive care unit (NICU) admission, readmission, and also higher neonatal mortality than term infants. However, late preterm infants have not been studied extensively, especially in developing countries.

Our aim in this study was to explore the birth rate, delivery mode, medical problems, requirement of respi- 
ratory support, and acute outcomes of late preterm infants in Zhejiang province located in eastern China.

\section{Methods}

Eleven tertiary hospitals which include about $70 \%$ of all neonatal nursery beds in the Zhejiang province, and serve a population of $\sim 39$ million were recruited. Nine nurseries were regional referral centers, admitting both inborn and outborn infants; one unit is the university women's hospital, accepted high-risk pregnant women with complications; another is the university children's hospital, served as a referral center for other units, providing the full range of NICU services, and includes cardiac surgery. Highrisk pregnancy or sicker infants were usually transferred to the university women's and children's hospital, so these two units were defined as high-risk centers, and the other nine centers were defined as relatively low-risk centers in this study.

All neonates with some medical problems admitted to the 11 units from January 2007 to December 2007 were enrolled in the study. An admission was defined as stay in the nursery for at least $24 \mathrm{~h}$ or death within $24 \mathrm{~h}$ of admission to the nursery. Infants who were withdrawn from medical care or transferred to another unit within $24 \mathrm{~h}$ of admission were also included. All babies were discharged from the 11 hospitals and none was retransferred back to a lower level nursery.

Chart review was performed on each late-preterm or term infant who was admitted to these 11 nurseries during the study period. Data were collected by trained staff, which included demographic characteristics, mode of delivery, main medical problems, respiratory support during hospitalization, and acute outcomes. Data collection was approved by the Ethics Committee of Children's Hospital, Zhejiang University, and adopted by each center. Data collection was supervised by the staff in Children's Hospital of Zhejiang University, which was responsible for quality assurance and correction of incomplete or ineligible records.

Gestational age was estimated from the first day of last menstrual period. All newborn infants were divided into three groups: early preterm infants $(<336 / 7$ weeks' gestation), late preterm $(34 \% / 7-36 \% / 7$ weeks) and term infants born $>37 \%$ weeks' gestation.

Medical problem was defined as a newborn at risk or at need of some intensive care or close observations. The diagnosis of respiratory distress syndrome (RDS) was established on the basis of clinical signs, i.e., tachypnea, retractions, nasal flaring, grunting and cyanosis, and the classic radiographic findings of transient tachypnea of newborn (TTN) or the reticulogranular pattern of RDS, and/or oxygen requirement for at least $2 \mathrm{~h}$.

Hyperbilirubinemia was defined according to serum bilirubin concentration or transcutaneous bilirubin level. Hypoglycemia was defined as blood sugar concentration $<2.2 \mathrm{mmol} / \mathrm{L}$ (40 mg/ $\mathrm{dL}$ ).

Respiratory support was defined as infants requiring nasal continuous positive airway pressure (nCPAP) or mechanical ventilation. Acute outcomes include infants who recovered or improved after the treatment, and discharged home based on physician's decision. Withdrawal from treatment means that the infant did not complete treatment for some reason, and was discharged home by the decision of the parents.
The primary goal of this study was to provide descriptive statistics of the study subjects and their outcomes. Univariate analyses on categorical data were performed by a 2-tailed Pearson $\chi^{2}$-test. For significant associations, the maximum likelihood estimate of the conditional odds ratio (OR) and 95\% confidence interval $(\mathrm{Cl})$ are reported. All statistical analyses were performed by SPSS 13.0 software (SPSS, Chicago, IL). P $<0.05$ was considered statistically significant.

\section{Results}

From January 2007 to December 2007, there were 44,362 infants born at the 11 hospitals, 1219 (2.7\%) infants were early preterm and 2742 (6.2\%) infants were late preterm. The overall preterm birth rate was $8.9 \%$. A total of 25,830 infants $(58.2 \%)$ were delivered by cesarean section. Among the 11 hospitals, the highest cesarean rate was $75.6 \%$ and the lowest $42.3 \%$ (Figure 1). Late preterm infants had the highest cesarean birth rate (64.9\%, $\mathrm{P}<0.05)$. For early preterm and term infants, the rate of cesarean section was $50.3 \%$ and $58.0 \%$, respectively.

During the study period, there were 10,537 infant admissions to the nurseries due to some medical problems. Late preterm infants accounted for $19.3 \%$ of all the admissions $(n=2032), 63.8 \%$ of whom were delivered by cesarean section, $89.2 \%$ were admitted at $<72$ h, $70.1 \%$ were inborn. Compared with term infants, more late preterm admissions were following cesarean births, inborn or admitted at $<3$ days of age (Table 1 ).

For late preterm admissions, respiratory distress $(n=856,42.1 \%)$ was the most common medical problem. Hyperbilirubinemia (17.6\%), hypoglycemia $(8.7 \%)$ and sepsis $(5.9 \%)$ were also common presentations on

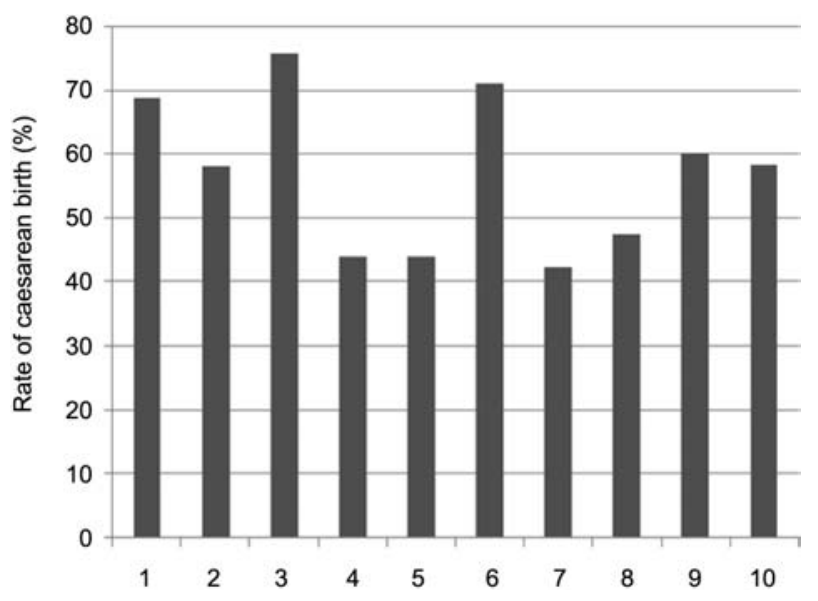

Figure 1 The rate of cesarean birth in 10 different hospitals (one hospital only accept outborn infant). 
admission (Table 2). The main primary diagnosis of respiratory distress consisted by pneumonia (39.5\%), TTN (22.5\%), RDS (19.0\%), blood or massive amniotic fluid aspiration (14.3\%) (Table 3).

More late preterm infants were supported with nCPAP $(21.4 \%$ vs. $11.6 \%, \mathrm{P}=0.000)$ or mechanical ventilation (15.4\% vs. $11.0 \%, \mathrm{P}=0.002)$ for respiratory distress (Table 4).

A total of $16(0.8 \%)$ late preterm admissions died, the in-hospital mortality was higher than that of term. Compared to term infants, less late preterm infants completed treatment $(87.5 \%$ vs. $89.3, P=0.02)$ and more late preterm infants were transferred to referral centers $(2.0 \%$ vs. $1.3 \%, P=0.035$ ) (Table 5).

The in-hospital mortality in high-risk centers and relatively low-risk centers was $1.0 \%$ and $0.7 \%$, respectively $(P=0.435)$. Difference between high-risk centers and relatively low-risk centers are shown in Table 6.

\section{Discussion}

Zhejiang province is located in the east coast of China and is one of the most developed provinces with a population of 51 million with about 500,000 births annually. In our study, the birth rate of late preterm infants in 2007 was $6.2 \%$, so the estimated annual late preterm births were 30,000 .

In today's China, both national and regional vital statistics still lack a detailed picture of perinatal care. However, data reported by several western countries $[3,6]$ indicate an increase of late preterm births, greater than the overall preterm birth rate. The reasons for the increase in late preterm births might include increasing proportion of pregnant women $>35$ years of age, multiple gestations due to reproductive technologies and more medically indicated deliveries secondary to better surveillance of the mother and the fetus [7]. Because late

Table 1 Demographics of nursery admissions.

\begin{tabular}{lrlll}
\hline & Early preterm & Late preterm & Term & All \\
\hline $\mathrm{n} \mathrm{( \% )}$ & $1538(14.6 \%)$ & $2032(19.3 \%)$ & $6967(66.1 \%)$ & $10537(100 \%)$ \\
Cesarean section, $\mathrm{n}(\%)$ & $781(50.8 \%)$ & $1297(63.8 \%)^{\star, * \star}$ & $3666(52.6 \%)$ & $5744(54.5 \%)$ \\
Admitted at age $\leq 3$ days, $\mathrm{n}(\%)$ & $1456(94.7 \%)$ & $1813(89.2 \%)^{\star, * \star}$ & $4245(60.9 \%)$ & $7514(71.3 \%)$ \\
Inborn, $\mathrm{n}(\%)$ & $1026(66.7 \%)$ & $1425(70.1 \%)^{\star, * \star}$ & $4135(59.4 \%)$ & $6586(62.5 \%)$ \\
\hline
\end{tabular}

${ }^{*} \mathrm{P}<0.05$ compared with early preterm infants.

${ }^{* *} \mathrm{P}<0.05$ compared with term infants.

Table 2 Main medical problems of late preterm and term nursery admissions.

\begin{tabular}{lccrrr}
\hline & $\begin{array}{l}\text { Late preterm infants } \\
(\mathrm{n}=2032)\end{array}$ & $\begin{array}{l}\text { Term infants } \\
(\mathrm{n}=6967)\end{array}$ & \multicolumn{1}{c}{$\chi^{2}$} & P-value & OR (95\% Cl) \\
\hline Respiratory distress, $\mathrm{n}(\%)$ & $856(42.1 \%)$ & $1773(25.4 \%)$ & 211.59 & 0.000 & $2.132(1.923-2.364)$ \\
HIE, $\mathrm{n}(\%)$ & $67(3.3 \%)$ & $167(2.4 \%)$ & 5.03 & 0.032 & $1.388(1.041-1.851)$ \\
Intracranial hemorrhage, $\mathrm{n}(\%)$ & $63(3.1 \%)$ & $86(1.2 \%)$ & 33.64 & 0.000 & $2.560(1.843-3.557)$ \\
Congenital heart disease, $\mathrm{n}(\%)$ & $75(3.7 \%)$ & $299(4.3 \%)$ & 1.43 & 0.255 & $0.855(0.660-1.106)$ \\
Congenital anomaly, $\mathrm{n}(\%)$ & $62(3.1 \%)$ & $198(2.8 \%)$ & 0.25 & 0.599 & $1.076(0.805-1.437)$ \\
Hyperbilirubinemia, $\mathrm{n}(\%)$ & $357(17.6 \%)$ & $1914(27.5 \%)$ & 81.78 & 0.000 & $0.563(0.496-0.638)$ \\
Sepsis, $\mathrm{n}(\%)$ & $119(5.9 \%)$ & $502(7.2 \%)$ & 4.46 & 0.037 & $0.801(0.652-0.985)$ \\
Hypoglycemia, $\mathrm{n}(\%)$ & $176(8.7 \%)$ & $204(2.9 \%)$ & 127.86 & 0.000 & $3.144(2.553 \sim 3.871)$ \\
\hline
\end{tabular}

$\mathrm{HIE}=$ hypoxic ischemic encephalopathy, $\mathrm{OR}=$ odds ratio, $\mathrm{Cl}=$ confidence interval.

Table 3 Causes of respiratory distress.

\begin{tabular}{lccrrr}
\hline & $\begin{array}{l}\text { Late preterm infants } \\
(\mathrm{n}=856)\end{array}$ & $\begin{array}{l}\text { Term infants } \\
(\mathrm{n}=1773)\end{array}$ & \multicolumn{1}{l}{$\chi^{2}$} & P-value & OR (95\% Cl) \\
\hline RDS, $\mathrm{n}(\%)$ & $163(19.0 \%)$ & $77(4.3 \%)$ & 150.36 & 0.000 & $5.181(3.896 \sim 6.890)$ \\
TTN, n (\%) & $193(22.5 \%)$ & $235(13.3 \%)$ & 36.57 & 0.000 & $1.905(1.543 \sim 2.353)$ \\
MAS, n (\%) & $17(2.0 \%)$ & $141(8.0 \%)$ & 36.38 & 0.000 & $0.235(0.141 \sim 0.391)$ \\
Blood or massive amniotic & $122(14.3 \%)$ & $450(25.4 \%)$ & 42.00 & 0.000 & $0.489(0.392 \sim 0.609)$ \\
fluid aspiration, n (\%) & & & & \\
Pneumothorax, $\mathrm{n}(\%)$ & $23(2.7 \%)$ & $66(3.7 \%)$ & 1.89 & 0.205 & $0.714(0.441 \sim 1.156)$ \\
Pneumonia, $\mathrm{n}(\%)$ & $338(39.5 \%)$ & $804(45.4 \%)$ & 8.07 & 0.005 & $0.786(0.666 \sim 0.928)$ \\
\hline
\end{tabular}

$\mathrm{RDS}=$ respiratory distress syndrome, $\mathrm{TTN}=$ transient tachypnea of newborn, MAS $=$ meconium aspiration syndrome, $\mathrm{OR}=\mathrm{odds}$ ratio, $\mathrm{Cl}=$ confidence interval. 
Table 4 Respiratory support required by infants with respiratory distress.

\begin{tabular}{lllll}
\hline & $\begin{array}{l}\text { Late preterm infants } \\
(\mathrm{n}=856)\end{array}$ & $\begin{array}{l}\text { Term infants } \\
(\mathrm{n}=1773)\end{array}$ & $\chi^{2}$ & P-value \\
\hline Mechanical ventilation, $\mathrm{n}(\%)$ & $132(15.4 \%)$ & $195(11.0 \%)$ & 10.37 & 0.002 \\
nCPAP, $\mathrm{n}(\%)$ & $183(21.4 \%)$ & $205(11.6 \%)$ & 44.22 & 0.000 \\
\hline
\end{tabular}

$\mathrm{nCPAP}=$ nasal continuous positive airway pressure.

Table 5 Acute clinical outcomes.

\begin{tabular}{lccrr}
\hline & $\begin{array}{l}\text { Late preterm infants } \\
(\mathrm{n}=2032)\end{array}$ & $\begin{array}{l}\text { Term infants } \\
(\mathrm{n}=6967)\end{array}$ & $\chi^{2}$ & P-value \\
\hline Complete treatment, $\mathrm{n}(\%)$ & $1777(87.5 \%)$ & $6224(89.3 \%)$ & 5.67 & 0.020 \\
Withdrawal of medical care, $\mathrm{n}(\%)$ & $199(9.8 \%)$ & $623(8.9 \%)$ & 1.37 & 0.238 \\
Transferred to referral center, $\mathrm{n}(\%)$ & $40(2.0 \%)$ & $91(1.3 \%)$ & 4.81 & 0.035 \\
In-hospital mortality, $\mathrm{n}(\%)$ & $16(0.8 \%)$ & $29(0.4 \%)$ & 4.36 & 0.048 \\
\hline
\end{tabular}

Table 6 Comparison of the main clinical data from high-risk centers and relatively low-risk centers.

\begin{tabular}{lccrrr}
\hline & $\begin{array}{l}\text { High-risk centers } \\
(\mathrm{n}=696)\end{array}$ & $\begin{array}{l}\text { Relative low-risk } \\
\text { centers }(\mathrm{n}=1336)\end{array}$ & $\chi^{2}$ & P-value & OR (95\% Cl) \\
\hline RDS, $\mathrm{n}(\%)$ & $87(12.5 \%)$ & $76(5.7 \%)$ & 28.78 & 0.000 & $2.368(1.715-3.270)$ \\
TTN, $\mathrm{n}(\%)$ & $112(16.1 \%)$ & $81(6.1 \%)$ & 53.55 & 0.000 & $2.971(2.196-4.020)$ \\
HIE, $\mathrm{n}(\%)$ & $19(2.7 \%)$ & $48(3.6 \%)$ & 1.07 & 0.360 & $0.753(0.439-1.291)$ \\
Congenital heart disease, $\mathrm{n}(\%)$ & $28(4.0 \%)$ & $47(3.5 \%)$ & 0.33 & 0.620 & $1.150(0.713-1.852)$ \\
Congenital anomaly, $\mathrm{n}(\%)$ & $34(4.9 \%)$ & $28(2.1 \%)$ & 12.04 & 0.001 & $2.399(1.442-3.991)$ \\
Hyperbilirubinemia, $\mathrm{n}(\%)$ & $76(10.9 \%)$ & $281(21.0 \%)$ & 32.32 & 0.000 & $0.460(0.351-0.604)$ \\
Sepsis, $\mathrm{n}(\%)$ & $62(8.9 \%)$ & $57(4.3 \%)$ & 17.89 & 0.000 & $2.194(1.513-3.183)$ \\
Hypoglycemia, $\mathrm{n}(\%)$ & $94(13.5 \%)$ & $82(6.1 \%)$ & 31.40 & 0.000 & $2.388(1.748-3.261)$ \\
Mechanical ventilation, $\mathrm{n}(\%)$ & $50(7.2 \%)$ & $82(6.1 \%)$ & 0.83 & 0.393 & $1.184(0.822-1.704)$ \\
nCPAP, n (\%) & $88(12.6 \%)$ & $95(7.1 \%)$ & 17.10 & 0.000 & $1.891(1.393-2.567)$ \\
Complete treatment, $\mathrm{n}(\%)$ & $593(85.2 \%)$ & $1184(88.6 \%)$ & 147.48 & 0.000 & $2.013(1.795-2.256)$ \\
Withdrawal of medical care, $\mathrm{n}(\%)$ & $74(10.6 \%)$ & $125(9.4 \%)$ & 0.843 & 0.387 & $1.153(0.851-1.561)$ \\
In-hospital mortality, $\mathrm{n}(\%)$ & $7(1.0 \%)$ & $9(0.7 \%)$ & 0.646 & 0.435 & $1.498(0.555-4.040)$ \\
\hline
\end{tabular}

$\mathrm{RDS}=$ respiratory distress syndrome, $\mathrm{TTN}=$ transient tachypnea of newborn, $\mathrm{nCPAP}=$ nasal continuous positive airway pressure, $\mathrm{HIE}=$ hypoxic ischemic encephalopathy, $\mathrm{OR}=$ odds ratio, $\mathrm{Cl}=$ confidence interval.

preterm infants comprise a large proportion of preterm infants, even a small increase in their birth rate might have a significant impact on the distribution of health care resources.

In a review article by Raju et al. [7], the rate of cesarean section was around 20\%-30\% in North America, however, the rate in China was reported as high as $40 \%-60 \%[1,11]$. Zhang et al. [11] reported in 2008 that cesarean section on maternal request has an important impact on the high rate of cesarean births. In our study, the overall cesarean birth rate was $58.2 \%$, and for late preterm infants this number was even higher (64.9\%). Reasons for the extremely high cesarean birth rate compared to other parts of the world are not clear and need further study.

One-fifth of our nursery admissions were for late preterm infants and respiratory distress was the most common medical problem at admission with pneumonia, RDS and TTN comprising nearly $80 \%$ of the total. Compared with term infants, the rate of RDS and TTN is higher in late preterm infants. Our data showed a relatively high incidence of pneumonia which might be due to the lack of strict criteria to define TTN, RDS, mild meconium aspiration and pneumonia in some participating nurseries, resulting in the possibility of overlap among these disorders. Another explanation is that for the $10 \%$ of the late preterm infants who were admitted after 3 days of age, pneumonia is the most common diagnosis.

The high cesarean section rate also contributes to the high incidence of respiratory distress. As several authors $[4,8,10]$ have reported, it might be possible that cesarean delivery is associated with increased risk of RDS and TTN. However, the rate of elective cesarean section in this study is not available.

Hypoglycemia and intracranial hemorrhage unsurprisingly occur more often in late preterm infants. In contrast, hyperbilirubinemia and sepsis seem to be unusually more common in term infants. There might be two explanations: about $40 \%$ of the term infants admitted to NICU 
were outborn and/or admitted at age $\geq 3$ days. For the "old" outborn term infants, the common reasons for admission were community acquired pneumonia, sepsis, or hyperbilirubinemia. Additionally, late preterm infants stay in hospital for a longer time than term infants (usually 5 days for vaginal delivery and 7 days for cesarean section), so the bilirubin level of late preterm infants is monitored diligently by pediatricians, and the chance to get antibiotics is also very high. As a result, the incidence of bilirubinemia and sepsis in late preterm infants was lower.

Compared with term infants, more late preterm infants with respiratory distress were diagnosed with RDS and TTN. Also more late preterm infants required nCPAP or ventilatory support, which suggests that the respiratory problems of late preterm infants were more severe. Compared to relatively low-risk centers, significantly more infants were admitted to high-risk centers for RDS or TTN (12.5\% vs. $5.7 \%$, and $16.1 \%$ vs. $6.1 \%)$. The use of nCPAP is almost twice as often in the high-risk centers, but no difference exists for requirement of mechanical ventilation, which indicates that many infants delivered by cesarean section only developed mild or moderate respiratory distress resolved with nCPAP.

We also described the frequencies of withdrawal medical care. There are two reasons for this. First is that some parents are concerned about the long-term sequela for some severe medical conditions, such as brain injury or congenital anomaly. The other is the economic embarrassment due to low income or lack of public health insurance, so when the infant improves, the parents decide to discharge it from hospital. In Zhejiang province, there is $>10$ million moving labor force, most of whom coming from rural area of other provinces, low income class without or with limited health insurance. It is clear that late preterm infants had higher in-hospital mortality than term infants. Unfortunately, we did not follow the infants who withdrew from medical care.

In China, late preterm infants are the group who has been ignored by health care givers for a long time. The health system in China is significantly different from developed countries, so the epidemiologic data reported by these countries are not appropriate. Due to the very high cesarean birth rate, our late preterm infants may face more medical complications. This large epidemiologic report provides the first detailed clinical profile of late preterm infants in Chinese. We believe this report reflects the epidemiology of late preterm infants in Zhejiang province, and also can represent the status in east China. In our study, there are some limitations. The first is that we only collected data from tertiary hospitals: this may introduce a selection bias. The second is that all the data were collected from individual hospital retrospectively, not from a standardized database, so that some important information was missing such as the incidence of elective cesarean section, maternal age, indications of cesarean birth. A prospective trial to assess the impact of high cesarean birth rate on the overall health care system is ongoing.

\section{References}

[1] Cheng YM, Yuan W, Cai WD, Zhang WM, Wang TY, Wang $Y$, et al. Study on the occurrence of cesarean section (CS) and factors related to CS in China. Zhonghua Liu Xing Bing Xue Za Zhi. 2003;24:893-6.

[2] Clark $\mathrm{RH}$. The epidemiology of respiratory failure in neonates born at an estimated gestational age of 34 weeks or more. J Perinatol. 2005;25:251-7.

[3] Davidoff MJ, Dias T, Damus K, Russell R, Bettegowda VR, Dolan $\mathrm{S}$, et al. Changes in the gestational age distribution among U.S. singleton births: impact on rates of late preterm birth, 1992-2002. Semin Perinatol. 2006;30:8-15.

[4] Hansen AK, Wisborg K, Uldbjerg N, Henriksen TB. Risk of respiratory morbidity in term infants delivered by elective Cesarean section: cohort study. Br Med J. 2008;336:85-7.

[5] Kramer MS, Demissie K, Yang H, Platt RW, Sauve R, Liston $\mathrm{R}$. The contribution of mild and moderate preterm birth to infant mortality. J Am Med Assoc. 2000;284:843-9.

[6] Martin JA, Hamilton BE, Sutton PD, Ventura SJ, Menacker F, Munson ML. Birth: final data for 2003. Natl Vital Stat Rep. 2005,54:1-116.

[7] Raju TNK. Epidemiology of late preterm (near-term) births. Clin Perinatol. 2006;33:751-63.

[8] Roth-Kleiner M, Wagner BP, Bachmann D, Pfenninger J. Respiratory distress syndrome in near-term babies after cesarean section. Swiss Med Wkly. 2003;133:283-8.

[9] Wang ML, Dorer DJ, Fleming MP, Catlin EA. Clinical outcomes of near-term infants. Pediatrics. 2004;114:372-6.

[10] Zanardo V, Simbi AK, Franzoi M, Soldà G, Salvadori A, Trevisanuto $D$. Neonatal respiratory morbidity risk and mode of delivery at term: influence of timing of elective cesarean delivery. Acta Paediatr. 2004;93:643-7.

[11] Zhang J, Liu Y, Meikle S, Zheng J, Sun W, Li Z. Cesarean delivery on maternal request in Southeast China. Obstet Gynecol. 2008;111:1077-82.

The authors stated that there are no conflicts of interest regarding the publication of this article.

Received February 11, 2009. Revised April 14, 2009. Accepted May 28, 2009. Previously published online July 10, 2009. 